\title{
Direct Observation of Intrachain Hydrogen Bonds in Aqueous Hyaluronan
}

\author{
Giulia Giubertoni, ${ }^{\circledR}$ Gijsje H. Koenderink, ${ }^{\circledR}$ and Huib J. Bakker*(우 \\ AMOLF, Science Park 104, 1098 XG Amsterdam, The Netherlands \\ Supporting Information
}

\begin{abstract}
We use two-dimensional infrared spectroscopy to study the interactions between the amide and carboxylate anion groups of hyaluronan polymers at neutral $\mathrm{pH}$. The spectra reveal the presence of intrachain hydrogen bonds between the amide and carboxylate anion groups in aqueous solution. We determine the relative orientation of the amide and carboxylate anion groups when forming this hydrogen bond and quantify the fraction of amide groups that participate in hydrogen bonding. We find that a variation of the $\mathrm{pH}$ and/or temperature has a negligible effect on this fraction, whereas the persistence length of the hyaluronan chains and the associated viscosity of hyaluronan solutions are known to change significantly. We conclude that the hydrogen bonding between the amide and carboxylate anion groups does not significantly contribute to the chain rigidity of hyaluronan polymers.
\end{abstract}

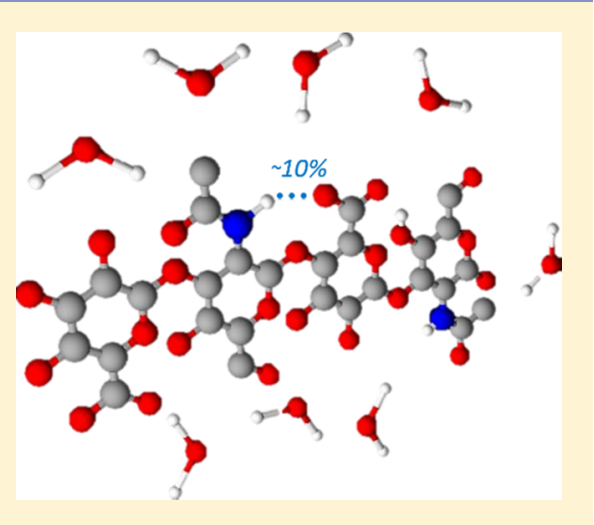

\section{INTRODUCTION}

Hyaluronan is a natural polysaccharide in the human body with important biological functionalities and, accordingly, also has many biomedical applications. ${ }^{1,2}$ Since the 1940s, hyaluronan has been the subject of numerous studies, investigating its physicochemical properties and their role in biological functions such as stem cell fate determination and tissue lubrication. $^{3-6}$ The viscoelastic properties of aqueous solutions of hyaluronan were found to strongly depend on the concentration and on the molecular weight of the polysaccharide, in accordance with semiflexible polymer models. ${ }^{7}$ The mechanical properties also strongly depend on the nature and concentration of added salts and the $\mathrm{pH}$ of the solution, ${ }^{2,8-10}$ a phenomenon that is much less well understood. The physicochemical conditions likely influence the conformation of hyaluronan polymers and their interactions, but direct experimental evidence is lacking.

Hyaluronan polymers show an exceptionally large bending rigidity under physiological conditions, with a persistence length between 4 and $7 \mathrm{~nm}$, corresponding to approximately four to seven monomers. ${ }^{11,12}$ This feature has been interpreted as a strong indication for the presence of intramolecular hydrogen bonds, which locally stiffen the chain by limiting the rotation of the disaccharide units around the glycosidic bonds. ${ }^{13}$ Indeed, for solutions of hyaluronan tetrasaccharides in dimethyl sulfoxide, strong evidence for the existence of specific intrachain hydrogen bonds between the amide and the carboxylate anion groups has been found by means of NMR. ${ }^{14}$ Based on this observation, it was proposed that the secondary conformations of long chains of hyaluronan polymers, such as helical structures, could be stabilized by intrachain hydrogen bonds between amide and carboxylate groups. ${ }^{14}$
Several molecular dynamics studies indicated that direct hydrogen bonds between amide and carboxylate anion groups should be formed also in aqueous solution under physiological conditions. ${ }^{15}$ However, some other NMR and molecular dynamics studies suggested that the probability of forming this type of hydrogen bond is smaller than $10 \% .{ }^{16-18}$ In fact, up to now, there has been no direct experimental evidence for the existence of intrachain hydrogen bonds between the amide and carboxylate anion groups.

Two-dimensional infrared (2D-IR) spectroscopy is one of the few techniques capable of directly probing interactions such as hydrogen bonding at the molecular scale in aqueous (bio)molecular solutions. ${ }^{19}$ Similar to two-dimensional nuclear magnetic resonance (2D-NMR), 2D-IR can resolve molecular couplings and dynamics but at a much shorter time scale. Molecular interactions such as hydrogen bonds or electrostatic forces usually change on a time scale of picoseconds, ${ }^{20}$ and 2DIR is ideally suited to take snapshots of these interactions with subpicosecond time resolution. Here, we apply polarizationresolved 2D-IR spectroscopy to study the interaction and relative orientation of the amide and carboxylate anion groups of hyaluronan in aqueous solutions and as a function of ionic strength and temperature.

\section{EXPERIMENTAL METHODS}

Sample Preparation. Aqueous hyaluronan solutions were prepared in a glass vial by mixing heavy water, $\mathrm{NaCl}(0.15 \mathrm{M})$, and $\mathrm{NaOD}$ to achieve different $\mathrm{pH}$ values. All of these chemicals were provided by Sigma-Aldrich. A specific amount

Received: July 8, 2019

Revised: August 23, 2019

Published: September 3, 2019 
of hyaluronic acid sodium salt in powder form from SigmaAldrich $\left(M_{\mathrm{w}}>1 \mathrm{MDa}\right)$ or from Lifecore Biomedical $\left(M_{\mathrm{w}} \sim\right.$ $100 \mathrm{kDa}$ ) was added to the solution to reach a final concentration ranging from 15 to $20 \mathrm{mg} / \mathrm{mL}$. N-Acetylglucosamine in powder form was provided by Sigma-Aldrich. The samples were left to equilibrate at room temperature for at least $24 \mathrm{~h}$ before measuring. Samples were stored for at most 1 week at a temperature of $4{ }^{\circ} \mathrm{C}$, to slow down hydrolytic degradation process. $^{21}$

Fourier Transform Infrared (FTIR). All linear absorption measurements were performed using a Bruker Vertex $80 \mathrm{v}$ FTIR spectrometer equipped with a liquid-nitrogen-cooled mercury-cadmium-telluride (MCT) detector. The spectra were recorded under a nitrogen atmosphere with a resolution of 3 $\mathrm{cm}^{-1}$ using a standard sample cell with a path length of 100 $\mu \mathrm{m}$. Every spectrum represents an average of 100 scans. The reported spectra are corrected for the absorption of the solvent background.

2D-IR. We perform 2D-IR experiments with a homebuilt setup that has been described before. ${ }^{22}$ The excitation is performed with a pair of femtosecond mid-infrared pulses ( $\sim 100 \mathrm{fs}, 4 \mu \mathrm{J}$ per pulse). This excitation pulse pair induces transient absorption changes, which are monitored by a probe pulse $(\sim 100 \mathrm{fs}, 0.35 \mu \mathrm{J})$ that is delayed by a time $T_{\mathrm{w}}$. After transmission through the sample, the probe pulse is sent into an infrared spectrograph and detected with an infrared mercury-cadmium-telluride (MCT) detector array, thus yielding the transient absorption spectrum as a function of the probe frequency. The dependence of the transient absorption spectrum on the excitation frequency is determined by measuring transient spectra for many different delay times between the two excitation pulses. By Fourier transformation of these spectra, we obtain the dependence of the transient absorption spectrum on the excitation frequency. By plotting the transient absorption spectrum as a function of the excitation and the probing frequency, we obtain a twodimensional infrared (2D-IR) transient absorption spectrum for each delay time $T_{\mathrm{w}}$.

The femtosecond excitation pulses are centered at 1620 $\mathrm{cm}^{-1}$ and have a bandwidth of $200 \mathrm{~cm}^{-1}$. The probe pulse is centered at 1620 or $1480 \mathrm{~cm}^{-1}$ and also has a bandwidth of $200 \mathrm{~cm}^{-1}$. The spectra of the excitation and probe pulses are sufficiently broad to cover the $\nu=0$ to $\nu=1$ (bleach) and the $\nu$ $=1$ to $\nu=2$ (excited-state absorption, or esa) transitions of the antisymmetric vibration $\left(\nu_{\text {ant, } \mathrm{COO}^{-}}\right)$of the carboxylate anion group and the amide I/II vibrations $\left(\nu_{\mathrm{AM} . \mathrm{I}} / \nu_{\mathrm{AM} \text {.II }}\right)$. We measure transient spectra using probing pulses that are polarized parallel and perpendicular with respect to the polarization of the excitation pulses.

\section{RESULTS AND DISCUSSION}

Linear Infrared Spectroscopy. Hyaluronan is a linear polysaccharide whose basic structure is a repeating unit composed of $\mathrm{N}$-acetyl-glucosamine and glucuronic acid (the inset in Figure 1). Figure 1 shows the linear infrared spectra for protonated (HA) and deprotonated $\left(\mathrm{HA}^{-}\right)$hyaluronan in heavy water solutions at a concentration of $20 \mathrm{mg} / \mathrm{mL}$. The spectra of the two species are extracted by fitting linear infrared spectra measured for hyaluronan in different solutions with a $\mathrm{pH}$ ranging from 1.5 to 6.9 (Figure S1). The $\mathrm{HA}^{-}$infrared spectrum shows strong absorption bands centered around 1400 and $1600 \mathrm{~cm}^{-1}$ due to the molecular vibrations of the carboxylate anion: the symmetric stretching and antisymmetric

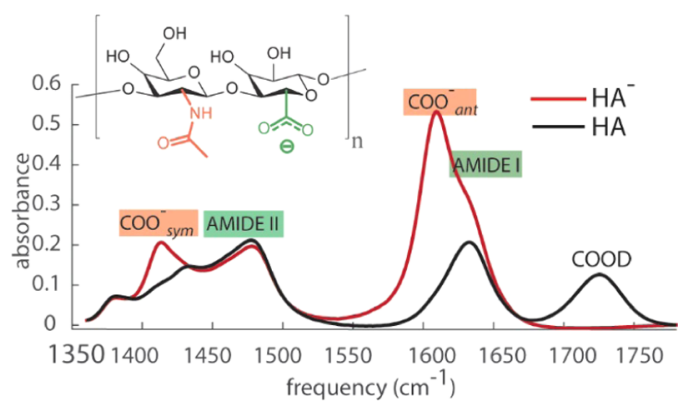

Figure 1. Linear infrared spectra of protonated $-\mathrm{COOH}(\mathrm{HA})$ and deprotonated $-\mathrm{COO}^{-}\left(\mathrm{HA}^{-}\right)$hyaluronan dissolved at a concentration of $20 \mathrm{mg} / \mathrm{mL}$ in heavy water. The linear spectra are obtained by decomposing measured linear spectra as explained in the Supporting Information. We observe that the main difference between the $\mathrm{HA}$ and $\mathrm{HA}^{-}$spectra is the presence of $-\mathrm{COO}^{-}$molecular vibrations at 1400 and $1600 \mathrm{~cm}^{-1}$. In the inset, a schematic of the molecular structure of the repeating units of hyaluronan is shown. The disaccharide contains $\mathrm{N}$-acetyl-glucosamine linked to glucuronate via a glycosidic bond.

stretching modes, respectively. Under acidic conditions, these bands give way to a new band at $1725 \mathrm{~cm}^{-1}$ representing the carbonyl stretch vibration of the carboxylic acid group. For both $\mathrm{HA}$ and $\mathrm{HA}^{-}$, we observe a band at $1633 \mathrm{~cm}^{-1}$, which corresponds to the amide I mode, and a band at around 1480 $\mathrm{cm}^{-1}$, corresponding to the amide II mode. ${ }^{23}$

Intramolecular Interaction between Amide and Carboxylate Anion Groups. Figure 2a shows the 2D-IR spectrum measured for a solution of hyaluronan at $20 \mathrm{mg} / \mathrm{mL}$ at $\mathrm{pH}=6.8$ at room temperature for a waiting time $T_{\mathrm{w}}$ of 0.7 ps. This spectrum represents the isotropic response, and it is obtained by adding the 2D-IR spectrum measured with parallel polarization to two times the 2D-IR spectrum measured with perpendicular polarization. ${ }^{19}$ We observe the presence of two pairs of diagonal peaks. Upon excitation at $1607 \mathrm{~cm}^{-1}$, we observe a strong bleach (blue-colored) and induced absorption (red-colored) peaks, representing the spectral response of the fundamental transition and the excited-state absorption of the antisymmetric stretching mode of the carboxylate anion. Similarly, we observe upon excitation at a pump frequency of $1633 \mathrm{~cm}^{-1}$ bleach and excited-state absorption signals arising from the excitation of the amide I vibration. In the 2D-IR spectrum, similarly to the linear infrared spectrum, the two vibrations strongly overlap. This spectral overlap obscures the off-diagonal regions of the $2 \mathrm{D}$-IR spectrum that contain information on the coupling of the different vibrations in the form of so-called cross-peak signals and thus on potential molecular interactions. To reduce the diagonal signals of the amide and carboxylate modes, we subtract the parallel 2D-IR spectrum from three times the perpendicular 2D-IR spectrum. $^{24}$ Nevertheless, in Figure $2 b$, we still observe a significant diagonal signal for the antisymmetric stretching mode and a signal corresponding to the $1 \rightarrow 2$ transition of this mode. It follows from the time-dependent anisotropy of these signals that the carboxylate anion rapidly reorients over a limited angle with a time constant of $\sim 250 \mathrm{fs}$ (see Figure S2 of the Supporting Information). In addition to the residual diagonal signal, we observe cross-peak signals for an excitation frequency of $1610 \mathrm{~cm}^{-1}$ and a probing frequency of $1640 \mathrm{~cm}^{-1}$ (upward cross-peak), and for an excitation frequency of 1640 $\mathrm{cm}^{-1}$ and a probing frequency of $1610 \mathrm{~cm}^{-1}$ (downward crosspeak). These off-diagonal features signal the presence of 


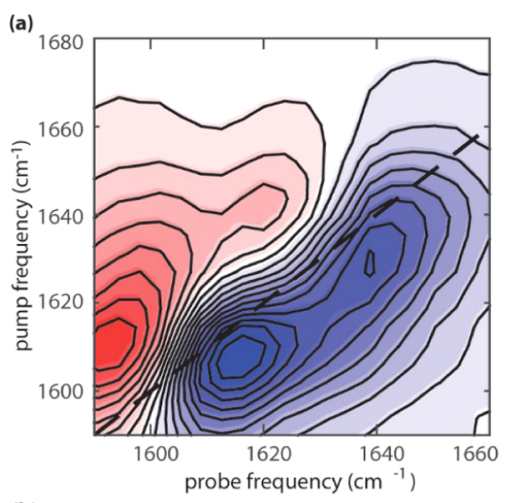

(b)
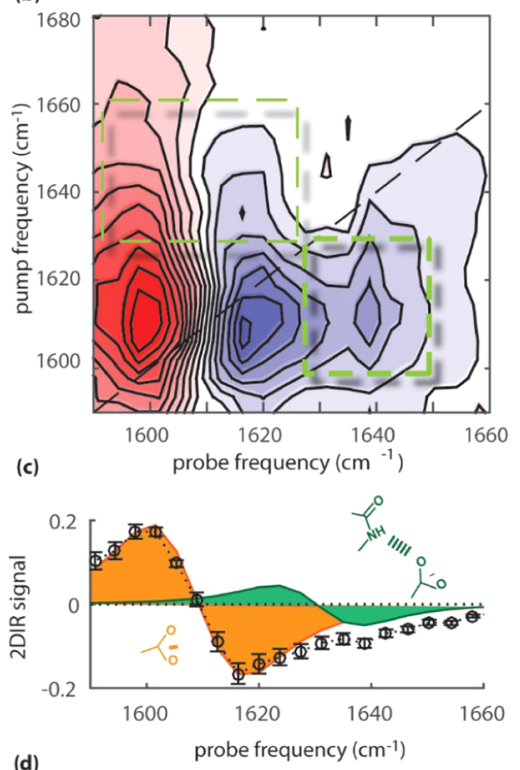

(d)

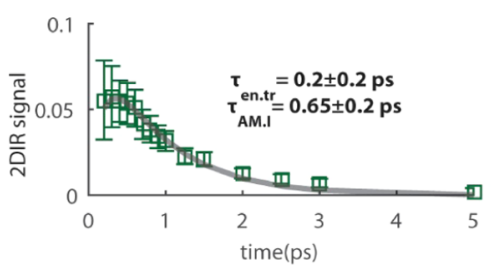

Figure 2. (a) Isotropic 2D-IR spectrum for a solution of $20 \mathrm{mg} / \mathrm{mL}$ hyaluronan in heavy water at $\mathrm{pH}=6.8$. (b) $2 \mathrm{D}$-IR spectrum obtained by subtracting the parallel signal from three times the perpendicular signal. The green rectangles indicate the off-diagonal regions, which reveal cross-peak signals arising from the vibrational coupling between amide I and $\mathrm{COO}^{-}$modes. (c) $2 \mathrm{D}$-IR absorption signal as a function of probe frequency obtained by averaging over the pump frequency range between 1600 and $1615 \mathrm{~cm}^{-1}$. The orange spectral signature represents the antisymmetric stretching vibration of the carboxylate anion group, and the green spectrum represents the cross-peak signal of this vibration and the amide I vibration (Figure S4). (d) Dependence of the cross-peak signal on the waiting time $T_{\mathrm{w}}$ between the probing pulses and the two excitation pulses (symbols), fitted to a double-exponential decay (grey line). The fit reveals a rise time constant $\left(t_{\text {en.tr }}\right)$ of $0.2 \pm 0.2 \mathrm{ps}$ and a decay time constant $\left(t_{\mathrm{AMI}}\right)$ of 0.6 \pm 0.2 ps.

vibrational coupling between the amide I vibration and antisymmetric stretch vibration of the carboxylate anion group.

To study the origin of the cross-peak, we measure the temporal dynamics of the upward cross-peak. It is difficult to study this dynamics for the downward cross-peak, as this signal is strongly obscured by the diagonal signal of the antisymmetric stretch vibration of the carboxylate anion. The cross-peak signal (excitation at $1614 \mathrm{~cm}^{-1}$, detection at 1637 $\mathrm{cm}^{-1}$ ) shows a rise followed by a slower decay (see Figure S3 of the Supporting Information), which indicates that it results from energy transfer between the excited antisymmetric stretching mode of $\mathrm{COO}^{-}$and the probed amide I vibration. Hence, the cross-peak signal is expected to have a similar spectral shape as the diagonal signal of the amide I vibration, showing a positive excited-state absorption signal and a negative bleaching signal.

Figure $2 \mathrm{c}$ shows the transient spectrum at $T_{\mathrm{w}}$ of $0.7 \mathrm{ps}$ obtained by averaging the $2 \mathrm{D}$-IR spectrum over the excitation frequency interval between 1605 and $1615 \mathrm{~cm}^{-1}$. In this figure, we also show the decomposition of the transient spectrum in spectral components that can be assigned to the diagonal signal of the antisymmetric stretching mode of the carboxylate anion and the cross-peak signal of the amide I vibration, represented by the orange and green spectral components, respectively. These spectral components are obtained from a global fit of the transient spectra to Lorentzian functions representing the excited-state absorption and bleaching signal of the antisymmetric stretching mode of $\mathrm{COO}^{-}$and Lorentzian functions defining the positive and negative parts of the cross-peak signal of the amide I vibration. We perform a global fit to the transient spectra measured at different delay times in which the widths, center frequencies, and amplitudes of the Lorentzians are global parameters (see the Supporting Information). We find that the bleach of the cross-peak has its maximum at 1637 $\mathrm{cm}^{-1}$.

In Figure 2d, we show the amplitude of the decomposed spectrum of the cross-peak as a function of delay. The crosspeak spectrum shows a fast rise, followed by a slower decay. We fit these data to a double-exponential function, with a rise time constant of $0.2 \pm 0.2$ ps and a decay time constant of 0.6 \pm 0.2 ps. We assign the decay to the vibrational relaxation of the amide I vibration.

The occurrence of fast energy transfer between the carboxylate anion and the amide group indicates that these groups are in close contact with each other. This molecular interaction must be of intramolecular origin, since there is no interchain aggregation in aqueous solution at neutral $\mathrm{pH}$, as evidenced by atomic force microscopy imaging and by rheology measurements. ${ }^{25}$ Hence, the fast rise of the crosspeak signal constitutes a strong indication that the amide and carboxylate groups interact via an intrachain hydrogen bond: $\mathrm{O}=\mathrm{C}-\mathrm{N}-\mathrm{H} \cdots \mathrm{O}-\mathrm{C}=\mathrm{O}^{-} \cdot{ }^{15}$

Relative Orientation of the Carboxylate Anion and Amide Groups. To study the molecular interaction between the amide and carboxylate anion groups in more detail, we also measured the coupling between the amide II vibration of the amide group and the antisymmetric stretch vibration of the carboxylate anion (Figure 3a). The amide II vibration absorbs at $1490 \mathrm{~cm}^{-1}$ and is mainly formed by a $\mathrm{C}-\mathrm{N}$ vibration. Its transition dipole moment is at an angle of $\sim 70^{\circ}$ with respect to the transition dipole moment of the amide I mode in heavy water solutions. $^{26,27}$ Figure 3 a shows the 2D-IR spectrum in the frequency region of the amide II spectral region, measured with a parallel polarization configuration of the excitation and probing pulses, at a waiting time $T_{\mathrm{w}}=0.3 \mathrm{ps}$. We observe a clear cross-peak signal for excitation frequencies between 1600 and $1640 \mathrm{~cm}^{-1}$ and probing frequencies around $1490 \mathrm{~cm}^{-1}$. This cross-peak signal shows an antidiagonally elongated line 

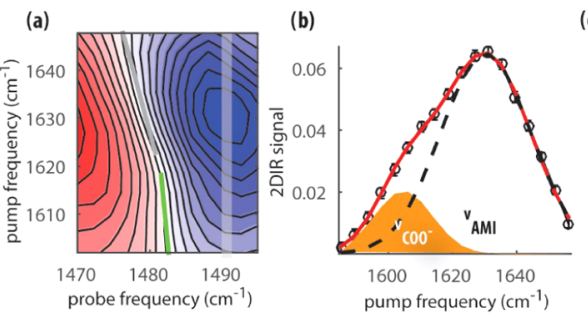

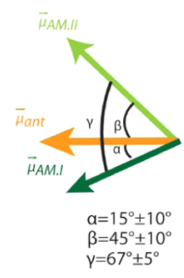

(d)

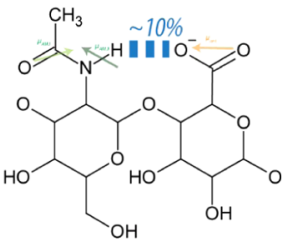

Figure 3. (a) 2D-IR spectrum measured in parallel polarization for a solution of hyaluronan at $\mathrm{pH}=6.8$ at a waiting time $T_{\mathrm{w}}=0.3$ ps. Gray and green lines represent the nodal lines. (b) Transient spectrum as a function of pump frequency obtained by averaging the $2 \mathrm{D}$-IR spectra over the probe frequency range between 1488 and $1492 \mathrm{~cm}^{-1}$, measured with a parallel polarization configuration of the pump and probe pulses. The orange Gaussian-shaped peak represents the cross-peak upon excitation of the antisymmetric stretching mode of the carboxylate anion group. The corresponding transient spectrum measured with a perpendicular polarization configuration of the pump and probe pulses is shown in Figure S6b. (c) Schematic representation of the relative orientation of the transition dipole moments of amide I $\left(\mu \rightarrow_{\text {AMI }}\right)$, amide II $\left(\mu \rightarrow_{\text {AMII }}\right)$, and antisymmetric stretching $\left(\mu-{ }_{\text {ant }}\right)$ vibrations upon the formation of a hydrogen bond between the amide and carboxylate anion groups. (d) Illustration of the intramolecular hydrogen bond between the amide and carboxylate anion groups in one single disaccharide unit.

shape, which is typical for the cross-peak of the amide I and amide II vibrations. ${ }^{26}$ We also observe that the nodal line slope is substantially larger for high excitation frequencies than for low excitation frequencies, which suggests the presence of an additional cross-peak signal at lower excitation frequencies, i.e., of the amide II vibration and the antisymmetric stretch vibration of the carboxylate anion. Indeed, this difference in the nodal line slope between high and low excitation frequencies is not observed in the 2D-IR spectrum of $N$ acetyl-glucosamine, which only exhibits a cross-peak between the amide I and amide II vibrations (Figure S5). In Figure 3b, we plot the parallel signal as a function of excitation frequency obtained by averaging over a narrow probe frequency interval between 1488 and $1492 \mathrm{~cm}^{-1}$ (Figure S6 shows the comparison between parallel and perpendicular signals). We observe that this signal shows a significant absorption between 1600 and $1615 \mathrm{~cm}^{-1}$, confirming the presence of a significant vibrational coupling between the amide II vibration and the antisymmetric stretch vibration of the carboxylate anion.

The polarization dependence of the 2D-IR measurements provides information on the angles between the transition dipole moments of the amide I mode (which is largely parallel to the $\mathrm{C}=\mathrm{O}$ bond of the amide group), the amide II mode (which is largely parallel to the $\mathrm{C}-\mathrm{N}$ bond), and the antisymmetric stretching vibration of the carboxylate anion group (which lies close to a line connecting the two oxygen atoms), as illustrated in Figure 3d. We thus decompose the parallel and perpendicular transient spectra obtained by pumping and probing in the amide I region (Figure S7). By measuring the ratio between the parallel and perpendicular signals of the cross-peak, we find an angle between the transition dipole moments of the amide $I$ and the antisymmetric stretching mode of the carboxylate anion of $15 \pm 10^{\circ}$.

The angle between the transition dipole moments of the amide I and of the amide II is expected to be $67 \pm 5^{\circ}$ (Figure $3 c)$. Hence, we expect that the transition dipole moments of the amide II and of the antisymmetric stretching vibrations will be at an angle of $\sim 50^{\circ}$, provided that all vibrations are in the same plane. By extracting the ratio between the parallel and perpendicular signals, measured for excitation frequencies between 1600 and $1615 \mathrm{~cm}^{-1}$ and for a detection frequency around $1490 \mathrm{~cm}^{-1}$, we find that the angle between the transition dipole moments of the amide II vibration and the antisymmetric stretch vibration of the carboxylate anion is 45 $\pm 10^{\circ}$.
The obtained angles between the transition dipole moments of the vibrations of the amide and the carboxylate anion groups indicate that the orientation of these groups is highly constrained, which points at the formation of a direct intrachain $\mathrm{O}=\mathrm{C}-\mathrm{N}-\mathrm{H} \cdots \mathrm{O}-\mathrm{C}=\mathrm{O}$ hydrogen bond. In this hydrogen bond, the hydrogen atom is located close to the line that connects the two oxygen atoms of the carboxylate anion (Figure 3d). Since the formation of this hydrogen bond causes a direct vibrational coupling between the amide I and the antisymmetric stretching modes, we can retrieve the fraction of amide and carboxylate anion groups that are bonded. We thus decompose the transient spectrum (shown in Figure S8) obtained from the isotropic 2D-IR spectrum into a diagonal signal $\Delta \alpha_{\text {iso }}^{\mathrm{COO}-}(t)$ of the $\nu_{\mathrm{COO}^{-}}$vibration and a $\Delta \alpha_{\text {iso }}^{\mathrm{cp}}(t)$ crosspeak signal. After the energy transfer is complete, we find $\frac{\Delta \alpha_{\text {iso }}^{\mathrm{cp}}}{\Delta \alpha_{\mathrm{iso}}^{\mathrm{COO}}} \sim 0.1$. This ratio provides information on the fraction $\left(f^{\text {nnt }}\right)$ of excited antisymmetric stretching vibrations of carboxylate anions that relax via energy transfer to the amide I vibration. We use $\frac{\Delta \alpha_{\text {iso }}^{\mathrm{cp}}}{\Delta \alpha_{\mathrm{iso}}^{\mathrm{COO}}}=f^{\text {ent }} \frac{\sigma_{\mathrm{AMI}}}{\sigma_{\mathrm{COO}^{-}}}$, where $\sigma_{\mathrm{AM} . \mathrm{I}}$ and $\sigma_{\mathrm{COO}^{-}}$ are the cross sections of the $\nu_{\mathrm{AM} \text {.I }}$ and $\nu_{\mathrm{COO}^{-}}$vibrations, respectively. The ratio $\frac{\sigma_{\mathrm{AMI}}}{\sigma_{\mathrm{COO}^{-}}}$is close to 1 (Figure S9), which implies that $10 \pm 5 \%$ of the antisymmetric $\nu_{\mathrm{COO}^{-}}$oscillators decays via energy transfer to the amide I vibration, as a consequence of the formation of a strong hydrogen bond between the carboxyl and amide groups.

Effect of the pH and Temperature. It has been observed that the stiffness of hyaluronan chains drops down drastically $(30-50 \%)$ when the $\mathrm{pH}$ is increased from neutral to alkaline. $^{28,29}$ To understand the effect of the $\mathrm{pH}$ on the hydrogen bond between the amide and carboxylate anion groups, we measured $2 \mathrm{D}$-IR spectra at different $\mathrm{pH}$ values and calculated the fractions of carboxylate groups that relax via energy transfer to the amide I vibration (Figure S10). In Figure $4 \mathrm{a}$, we show the fractions as a function of $\mathrm{pH}$. We observe that an increase in $\mathrm{pH}$ from 6.8 up to 13 does not lead to a change of the fraction of bonded carboxylate anion groups.

The fact that an increase of $\mathrm{pH}$ leads to a decrease in the stiffness of the hyaluronan polymers but not to a change of the fraction that relaxes via energy transfer indicates that the intrachain hydrogen bond between the carboxylate anion group and the amide group is not a major determinant of chain stiffness. This conclusion supports a hypothesis put forward in the literature that the main source of chain stiffness is formed 

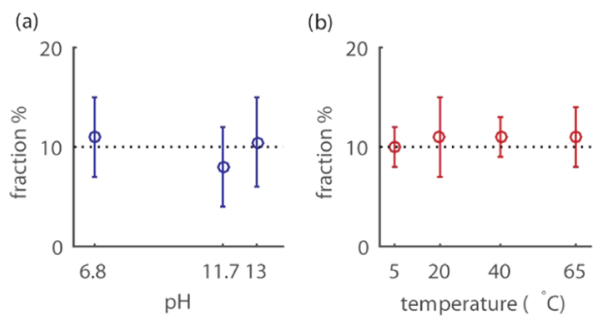

Figure 4. (a) Fractions of carboxylate groups that decay via energy transfer to the amide $\mathrm{I}$ at $\mathrm{pH}=6.8,11.7$, and 13. (b) Fractions of carboxylate groups that decay via energy transfer to the amide I at different temperatures. In both cases, the fractions are obtained by fitting the isotropic transient spectra (Figures S10 and S11).

by the hydrogen bonds/water bridges to the oxygen rings. ${ }^{7,28,30}$

It has also been reported that the viscosity of a hyaluronan solution drops significantly when the temperature is increased, likely also because of a reduction of the stiffness of the polymer chains. ${ }^{31-33}$ We measured 2D-IR spectra at different temperatures in the range between 5 and $65{ }^{\circ} \mathrm{C}$, which has been observed to lead to a decrease in the viscosity of $\sim 25 \% .^{32}$ In Figure $4 \mathrm{~b}$, we compare the fractions of bonded carboxylate as a function of temperature (Figure S11). Again, we do not observe a significant change in the fraction when varying the temperature in the range between 5 and $65^{\circ} \mathrm{C}$.

The absence of an effect of temperature on the fraction of intrachain $\mathrm{O}=\mathrm{C}-\mathrm{N}-\mathrm{H} \cdots \mathrm{O}-\mathrm{C}=\mathrm{O}$ hydrogen bonds between the amide and the carboxylate anion group is somewhat unexpected. If this fraction would have been dependent on a single hydrogen bond binding energy, and if this binding energy would not have been too high, the fraction of intrachain bonds would have been expected to decrease when the temperature is raised. However, the situation for the hyaluronan polymers is more complex, as is illustrated by the fact that only $\sim 10 \%$ of the intrachain $\mathrm{O}=\mathrm{C}-\mathrm{N}-\mathrm{H} \cdots \mathrm{O}-\mathrm{C}=$ $\mathrm{O}$ hydrogen bonds that could be formed are actually formed. This fraction relies on a detailed balance of solvation interactions, configurational entropy, and different intrachain interactions. With increasing temperature, all of these contributions to the free energy will change. With respect to the fraction intrachain $\mathrm{O}=\mathrm{C}-\mathrm{N}-\mathrm{H} \cdots \mathrm{O}-\mathrm{C}=\mathrm{O}$ hydrogen bonds, the temperature is observed to have little effect, suggesting that some effects may compensate each other. For instance, it could be that at low temperatures, the fraction of formed intrachain hydrogen bond is limited because a higher fraction would have a high associated energy penalty due to unfavorable solvation interactions. At higher temperatures, this energy penalty is reduced, which may compensate the tendency for the intrachain hydrogen bonds to break up, thus yielding a fraction of intrachain hydrogen bonds that is more or less temperature independent.

The independence of the connected fraction on temperature indicates that this interaction does not play an important role in the stiffness of the polymer chains, which does strongly depend on temperature. ${ }^{31-33}$ The observed decrease of the viscosity with temperature is thus likely caused by a decrease of the stiffness of the polymer chains induced by a reduction of hydrogen bonds/water bridges formed by the oxygen rings. $7,28,30$

\section{CONCLUSIONS}

We studied the intramolecular interactions of hyaluronan polymers in aqueous solution using two-dimensional infrared (2D-IR) spectroscopy. The measured 2D-IR spectra provided clear evidence for the existence of a direct intrachain $\mathrm{O}=\mathrm{C}-$ $\mathrm{N}-\mathrm{H} \cdots \mathrm{O}-\mathrm{C}=\mathrm{O}$ hydrogen bond between the amide and the carboxylate anion groups of the hyaluronic polymer units. From the amplitude of the cross-peak signal in the 2D-IR spectra, we concluded that this hydrogen bond is only formed for $\sim 10 \%$ of the amide groups. Our findings provide direct support for earlier conclusions that were based on NMR measurements and molecular dynamics simulations. ${ }^{16-18}$

By measuring 2D-IR spectra with different polarization configurations for the excitation and the probing pulses, we also determined the relative orientation of the transition dipole moment of the amide I and amide II vibrations with respect to the transition dipole moment of the antisymmetric stretch vibration of the carboxylate anion group, when these groups are connected by a direct hydrogen bond. We find these transition dipole moments to be at angles of $15 \pm 10$ and $45 \pm$ $10^{\circ}$, respectively. These angles strongly point at the molecular structure for the intrachain hydrogen bond that is illustrated in Figure $3 \mathrm{~d}$. In this structure, the hydrogen atom of the hydrogen-bond-donating $\mathrm{N}-\mathrm{H}$ group is positioned close to the line that connects the two oxygen atoms of the carboxylate anion group.

Variations in the solution $\mathrm{pH}$ or in the temperature did not change the fraction of antisymmetric carboxylate anion vibrations showing energy transfer to the amide group, whereas the persistence length of hyaluronan and the associated viscosity of hyaluronan solutions are known to change significantly. Therefore, our findings indicate that the intrachain hydrogen bond between the carboxylate anion group and the amide group does not play an important role in the stiffness of hyaluronan polymers.

\section{ASSOCIATED CONTENT}

\section{Supporting Information}

The Supporting Information is available free of charge on the ACS Publications website at DOI: 10.1021/acs.jpca.9b06462.

Methods; linear spectra; anisotropy decay; cross-peak dynamics; decompositions of the 2D-IR signal as a function of different polarization, temperature, and $\mathrm{pH}$; and 2D-IR spectrum of $n$-acetylgalactosamine (PDF)

\section{AUTHOR INFORMATION}

\section{Corresponding Author}

*E-mail: H.Bakker@amolf.nl.

ORCID

Giulia Giubertoni: 0000-0002-3417-4987

Gijsje H. Koenderink: 0000-0002-7823-8807

Huib J. Bakker: 0000-0003-1564-5314

\section{Notes}

The authors declare no competing financial interest.

\section{ACKNOWLEDGMENTS}

This work is part of the industrial partnership program Hybrid Soft Materials that is carried out under an agreement between Unilever Research and the Netherlands Organisation for Scientific Research (NWO). The authors wish to thank Dr. Ralf P. Richter (University of Leeds) for his advice. 


\section{REFERENCES}

(1) Kogan, G.; Šoltés, L.; Stern, R.; Gemeiner, P. Hyaluronic Acid: A Natural Biopolymer with a Broad Range of Biomedical and Industrial Applications. Biotechnol. Lett. 2007, 29, 17-25.

(2) Balazs, E. A.; Cui, J. The Story of Hyaluronan Putty. Bioact. Carbohydr. Diet. Fibre 2013, 2, 143-151.

(3) Singh, A.; Corvelli, M.; Unterman, S. A.; Wepasnick, K. A.; McDonnell, P.; Elisseeff, J. H. Enhanced Lubrication on Tissue and Biomaterial Surfaces through Peptide-Mediated Binding of Hyaluronic Acid. Nat. Mater. 2014, 13, 988-995.

(4) Raviv, U.; Giasson, S.; Kampf, N.; Gohy, J.-F.; Jérôme, R.; Klein, J. Lubrication by Charged Polymers. Nature 2003, 425, 163-165.

(5) Zhang, Z.; Christopher, G. F. The Nonlinear Viscoelasticity of Hyaluronic Acid and Its Role in Joint Lubrication. Soft Matter 2015, $11,2596-2603$.

(6) Chaudhuri, O.; Gu, L.; Klumpers, D.; Darnell, M.; Bencherif, S. A.; Weaver, J. C.; Huebsch, N.; Lee, H.; Lippens, E.; Duda, G. N.; et al. Hydrogels with Tunable Stress Relaxation Regulate Stem Cell Fate and Activity. Nat. Mater. 2016, 15, 326-334.

(7) Cowman, M. K.; Schmidt, T. A.; Raghavan, P.; Stecco, A. Viscoelastic Properties of Hyaluronan in Physiological Conditions. F1000Research 2015, 4, 622.

(8) Wu, S.; Ai, L.; Chen, J.; Kang, J.; Cui, S. W. Study of the Mechanism of Formation of Hyaluronan Putty at PH 2. 5: Part I. Experimental Measurements. Carbohydr. Polym. 2013, 98, 16771682.

(9) Gatej, I.; Popa, M.; Rinaudo, M. Role of the PH on Hyaluronan Behavior in Aqueous Solution. Biomacromolecules 2005, 6, 61-67.

(10) Gibbs, D. A.; Merrill, E. W.; Smith, K. A.; Balazs, E. A. Rheology of Hyaluronic Acid. Biopolymers 1968, 6, 777-791.

(11) Oelschlaeger, C.; Cota Pinto Coelho, M.; Willenbacher, N. Chain Flexibility and Dynamics of Polysaccharide Hyaluronan in Entangled Solutions: A High Frequency Rheology and Diffusing Wave Spectroscopy Study. Biomacromolecules 2013, 14, 3689-3696.

(12) Cleland, R. L. Viscometry and Sedimentation Equilibrium of Partially Hydrolyzed Hyaluronate: Comparison with Theoretical Models of Wormlike Chains. Biopolymers 1984, 23, 647-666.

(13) Almond, A.; Brass, A.; Sheehan, J. K. Oligosaccharides as Model Systems for Understanding Water-Biopolymer Interaction: Hydrated Dynamics of a Hyaluronan Decamer. J. Phys. Chem. B 2000, 104, 5634-5640.

(14) Scott, J. E.; Heatley, F.; Hull, W. E. Secondary Structure of Hyaluronate in Solution. A $1 \mathrm{H}$-n.m.r. Investigation at 300 and 500 $\mathrm{MHz}$ in [2H6]Dimethyl Sulphoxide Solution. Biochem. J. 1984, 220, $197-205$.

(15) Almond, A.; Sheehan, J. K.; Brass, A. Molecular Dynamics Simulations of the Two Disaccharides of Hyaluronan in Aqueous Solution. Glycobiology 1997, 7, 597-604.

(16) Almond, A.; DeAngelis, P. L.; Blundell, C. D. Hyaluronan: The Local Solution Conformation Determined by NMR and Computer Modeling Is Close to a Contracted Left-Handed 4-Fold Helix. J. Mol. Biol. 2006, 358, 1256-1269.

(17) Cowman, M. K.; Cozart, D.; Nakanishi, K.; Balazs, E. A. 1H NMR of Glycosaminoglycans and Hyaluronic Acid Oligosaccharides in Aqueous Solution: The Amide Proton Environment. Arch. Biochem. Biophys. 1984, 230, 203-212.

(18) Blundell, C. D.; Deangelis, P. L.; Almond, A. Hyaluronan: The Absence of Amide-Carboxylate Hydrogen Bonds and the Chain Conformation in Aqueous Solution Are Incompatible with Stable Secondary and Tertiary Structure Models. Biochem. J. 2006, 396, 487-498.

(19) Hamm, P.; Zanni, M. Concepts and Methods of 2D Infrared Spectroscopy; Cambridge University Press, 2011.

(20) Kolano, C.; Helbing, J.; Kozinski, M.; Sander, W.; Hamm, P. Watching Hydrogen-Bond Dynamics in a $\beta$-Turn by Transient TwoDimensional Infrared Spectroscopy. Nature 2006, 444, 469-472.

(21) Maleki, A.; Kjøniksen, A.-L.; Nyström, B. Effect of PH on the Behavior of Hyaluronic Acid in Dilute and Semidilute Aqueous Solutions. Macromol. Symp. 2008, 274, 131-140.
(22) Selig, O.; Siffels, R.; Rezus, Y. L. A. Ultrasensitive Ultrafast Vibrational Spectroscopy Employing the Near Field of Gold Nanoantennas. Phys. Rev. Lett. 2015, 114, No. 233004.

(23) Kovács, A.; Nyerges, B.; Izvekov, V. Vibrational Analysis of NAcetyl-Alpha-D-Glucosamine and Beta-D-Glucuronic Acid. J. Phys. Chem. B 2008, 112, 5728-5735.

(24) Woutersen, S.; Hamm, P. Isotope-Edited Two-Dimensional Vibrational Spectroscopy of Trialanine in Aqueous Solution. J. Chem. Phys. 2001, 114, 2727-2737.

(25) Giubertoni, G.; Burla, F.; Martinez-Torres, C.; Dutta, B.; Pletikapić, G.; Pelan, E. G.; Rezus, Y. L. A.; Koenderink, G. H.; Bakker, H. J. Molecular Origin of the Elastic State of Aqueous Hyaluronic Acid. J. Phys. Chem. B 2019, 123, 3043-3049.

(26) DeFlores, L. P.; Ganim, Z.; Ackley, S. F.; Chung, H. S.; Tokmakoff, A. The Anharmonic Vibrational Potential and Relaxation Pathways of the Amide I and II Modes of N-Methylacetamide. J. Phys. Chem. B 2006, 110, 18973-18980.

(27) Dijkstra, A. G.; La Cour Jansen, T.; Bloem, R.; Knoester, J. Vibrational Relaxation in Simulated Two-Dimensional Infrared Spectra of Two Amide Modes in Solution. J. Chem. Phys. 2007, 127, No. 194505.

(28) Mathews, M. B.; Decker, L. Conformation of Hyaluronate in Neutral and Alkaline Solutions. Biochim. Biophys. Acta 1977, 498, 259-263.

(29) Reed, C. E.; Li, X.; Reed, W. F. The Effects of PH on Hyaluronate as Observed by Light Scattering. Biopolymers 1989, 28, 1981-2000.

(30) Haxaire, K.; Braccini, I.; Milas, M.; Rinaudo, M.; Pérez, S. Conformational Behavior of Hyaluronan in Relation to Its Physical Properties as Probed by Molecular Modeling. Glycobiology 2000, 10, 587-594.

(31) Cleland, R. L. Effect of Temperature on the Limiting Viscosity Number of Hyaluronic Acid and Chondroitin 4-Sulfate. Biopolymers 1979, 18, 1821-1828.

(32) Fouissac, E.; Milas, M.; Rinaudo, M. Shear-Rate, Concentration, Molecular Weight, and Temperature Viscosity Dependences of Hyaluronate, a Wormlike Polyelectrolyte. Macromolecules 1993, 26, 6945-6951.

(33) Hoefting, J. M.; Cowman, M. K.; Matsuoka, S.; Balazs, E. Temperature Effect on the Dynamic Rheological Characteristics of Hyaluronan, Hylan a and Synvisc. Hyaluronan 2002, 103-108. 